\title{
Designing and Evaluating Digital Games for Frail Elderly Persons
}

\author{
Kathrin M. Gerling \\ Interaction Lab \\ University of Saskatchewan \\ 110 Science Place \\ S7N 5C9 Saskatoon, SK, Canada \\ +1306 9962327 \\ kathrin.gerling@usask.ca
}

\author{
Frank P. Schulte \\ General Psychology: Cognition \\ University of Duisburg-Essen \\ Forsthausweg 2 \\ 47057 Duisburg, Germany \\ +492033792519 \\ frank.schulte@uni-due.de
}

\author{
Maic Masuch \\ Entertainment Computing Group \\ University of Duisburg-Essen \\ Forsthausweg 2 \\ 47057 Duisburg, Germany \\ +492033791150 \\ maic.masuch@uni-due.de
}

\begin{abstract}
Game research increasingly addresses human factors of gaming. Though more and more seniors become players, game design for frail elderly has rarely been explored. This paper addresses game design for senior citizens experiencing age-related changes, especially cognitive and physical limitations. We introduce and evaluate the case study SilverPromenade, which is specifically aimed at providing institutionalized frail elderly with a new leisure activity. SilverPromenade allows players to go on virtual walks while accounting for special needs regarding game complexity, and simplistic interaction paradigms are provided using Nintendo's Wii Remote and the Balance Board for game control. Evaluation results suggest that despite age-related impairments, the game was generally accessible to elderly persons. Yet, differences between inexperienced and experienced players were observed which suggest that interaction problems may be reduced by engaging with games over a longer time. Findings also indicate that the engagement of elderly players transcends into their everyday life, and their social interaction increases among one another. Most importantly, the evaluation showed that games were perceived as enjoyable leisure activity, supporting the approach of applying digital games to raise the quality of life among frail elderly by fostering activity.
\end{abstract}

\section{Categories and Subject Descriptors}

H.5.m [Information Interfaces and Presentation (e.g., HCI)]: Miscellaneous; K.4.2 [Computers and Society]: Social Issues Assistive technologies for people with disabilities, Handicapped persons/special needs; K.8.0 [Personal Computing]: General Games.

\section{General Terms}

Measurement, Design, Experimentation, Human Factors.

\section{Keywords}

Game Design, Interface Design, Evaluation, Frail Elderly, Game Usability, Accessibility, Player Experience, Silver Gaming.

\begin{abstract}
Permission to make digital or hard copies of all or part of this work for personal or classroom use is granted without fee provided that copies are not made or distributed for profit or commercial advantage and that copies bear this notice and the full citation on the first page. To copy otherwise, to republish, to post on servers or to redistribute to lists, requires prior specific permission and/or a fee.
\end{abstract}

Short presentation, ACE'2011 - Lisbon, Portugal Copyright 2011 ACM 978-1-4503-0827-4/11/11 ...\$10.00.

\section{INTRODUCTION}

Due to the demographic transition, the elderly part of the population in western societies is growing, and many countries are experiencing increasing numbers of senior citizens living in nursing homes and community centers. Because of the prevalence of age-related changes and impairments in this group, it is often difficult to let the members participate in regular physical and social activities, leaving them with a limited set of leisure activities, frequently leading to loneliness, ultimately resulting in cognitive and physical decline.

Research results suggest that digital games may provide a means of engaging elderly persons in social and physical activities. Thereby, they offer the opportunity of fighting sedentary lifestyles among institutionalized elderly. Additionally, preliminary findings suggest that games can be applied to engage frail elderly in playful activities [28], thereby training their cognitive and physical abilities. Eventually, games may be used to increase the emotional well-being of senior citizens [14], contributing to their quality of life. Despite these promising first results, the design of gaming systems to entertain frail elderly persons living in nursing homes has not been addressed yet, as existing design approaches primarily focus on the creation of game-based systems for physical therapy [32]. However, this group might largely benefit from cognitive, physical and social activity which can be fostered through the engagement with digital games.

Based on existing considerations regarding the design of games for elderly persons as well as an analysis of age-related changes among seniors, the video game SilverPromenade was developed. It primarily addresses frail elderly players and was designed to be played in a nursing home environment. In this paper, we present details of the game design as well as results of an early evaluation of the playable prototype. The findings suggest that elderly persons living in nursing homes are generally interested in playing digital games and have an enjoyable gaming experience while engaging with SilverPromenade regardless of age-related changes. Based on these results, we identified three design opportunities for game designers wishing to address frail elderly.

\section{RELATED WORK}

Previous research has addressed the issue of digital game design for elderly persons from different perspectives, focusing on the creation of an enjoyable gaming experience for seniors, while trying to motivate them to engage in healthy behavior through engagement in play. 
Game concepts for elderly designed to foster social interaction and physical activity were investigated in several case studies. One prominent example is Walk2Win by Mubin et al. [20], in which players use their mobile phones to search the room for game artifacts. The authors suggest the creation of mobile games to engage senior citizens in mild exertion. The augmented dancing environment DanceAlong by Keyani et al. [15] also addresses the issue of physical activity among senior citizens, aiming to motivate seniors to engage in dancing along with popular movie scenes. Another study found that playing brain-training games on a portable game console had a positive correlation with reported fun for an elderly age group (compared to a younger control group) suggesting cognitive challenges provide a satisfying gameplay mechanic for the elderly [19]. Finally, the ElderGames project by Gamberini et al. [7] presents a tabletop gaming solution specifically designed to test and train cognitive abilities of elderly persons. Additionally, different game-based systems have been designed to encourage elderly persons to participate in physical therapy, for instance to support motor rehabilitation after stroke [2] or as balance training [32]. Likewise, the commercially available system SilverFit offers a variety of mini games and was designed to support physical therapy such as balance training among senior citizens [25].

Digital games for elderly persons have also been examined from different theoretical perspectives. Various game design guidelines and recommendations specifically addressing the issue of creating games for elderly audiences have been developed. Flores et al. focus on the creation of games for rehabilitation therapy [5] and recommend the implementation of interaction paradigms suitable for therapy, while Gamberini et al. [7] as well as Ijsselsteijn and colleagues [11] concentrate on games for entertainment and cognitive training purposes, particularly addressing the design of appropriate in-game challenges, e.g. regarding game complexity. Besides providing information on the design of game elements targeting elderly audiences, most recommendations also address game interface design and visual adaptability, frequently overlapping with considerations regarding the design of information systems for elderly persons in human-computer interaction [4]. Additional insights into appropriate themes and game contents for elderly have been provided by De Schutter and Vanden Abeele [25], discussing the creation of meaningful play for older adults.

Despite these considerations, only a few games have in fact been especially designed to entertain elderly audiences. One rare example is the senior-friendly online poker game TableTalk Poker by Shim et al. [26]. Also, the intergenerational game concept AgeInvaders by Khoo et al. [17] tries to engage elderly persons in playful activities. Only few research results regarding game design for frail elderly players are available, and existing work on game design for this audience primarily focuses on accessibility [8] and player performance [9]. Preliminary findings suggest that commercially available games are not accessible for frail persons [8], [10] as a variety of age-related changes and impairments has to be accounted for when designing for frail elderly [4]. On a general level, age affects human cognition and physical abilities: Common age-related changes include decrements in posture, balance and gait as well as fine motor skills [4], [29]. Aging also affects sensory processes such as vision and hearing [4], creating additional challenges for interaction designers as entertainment systems should account for this issue by providing visual and auditive adaptability [11]. Additionally, age-related changes may negatively influence cognitive processes such as short-term memory, attention and vigilance [4]. Finally, age-related diseases may influence the prevalence of impairments [29]. Consequently, this raises the need for games specifically designed for frail elderly audiences accounting for common age-related changes.

In this paper, we therefore address the issue of games designed to entertain senior citizens while specifically addressing the needs of institutionalized frail elderly.

\section{GAME: SILVER PROMENADE}

SilverPromenade is a video game which targets frail elderly persons living in full-care nursing homes. It aims to enable them to set out on virtual walks while allowing the players to pick up three different roles using the Nintendo Wii Remote and Wii Balance Board as input devices. The core concept of the game evolves around the combination of video material with mini games designed to engage the user in simple in-game activities. Two gaming scenarios for SilverPromenade were implemented using Microsoft XNA Game Studio 3.1.

\subsection{Exemplary Gaming Scenario}

The first gaming scenario features a walk in a forest, which is combined with two optional mini games in order to engage a maximum of three active users. Players are invited to pick up different roles to support each other and contribute to the game. Because of the core idea behind the scenario, the Balance Board can easily be implemented into the game: Depending on the regularity of the player's steps, a video of a walk through a park is shown (cf. Figure 2). To avoid the interruption of video playback, at least one step has to be conducted within the predefined time interval of five seconds.

The two mini games aim to engage players equipped with the Wii Remote: In the first game, a butterfly is repeatedly displayed in the top area of the screen moving from left to right. It has to be caught by pointing the remote at its position. Depending on the player's skill, butterfly speed and flying behavior are adjusted. In the second game, a rabbit is displayed at the bottom half of the screen and has to be counted by shaking the Wii Remote. It randomly peeks into the screen from the left or right side and quickly leaves again, which requires the player to react instantly to its appearance. Once the player has successfully counted a rabbit, it leaves the screen, returning at a later point of time.

In comparison with computer games for healthy, younger players, this scenario features rather simplistic challenges and a reduced level of complexity as recommended by prior work in the field of game design for frail elderly players [8]. It offers the possibility of virtually visiting the urban forest which is largely inaccessible to frail elderly persons due to the distance from the nursing home and bumpy roads which would easily put physically impaired persons in danger and generally strain frail elderly.

\subsection{Structural Elements of the Game Concept}

The following section gives a detailed description of the game concept based on theoretical approaches towards digital game design by Fullerton [6] and Adams [1].

\subsubsection{Players}

SilverPromenade is aimed at frail elderly persons suffering from age-related decrements in cognition and physical abilities with no or little previous gaming experience. It supports up to three active participants and encourages the participation of passive players who engage in play via comments on in-game actions [26]. To account for age-related changes, the following roles are available: 


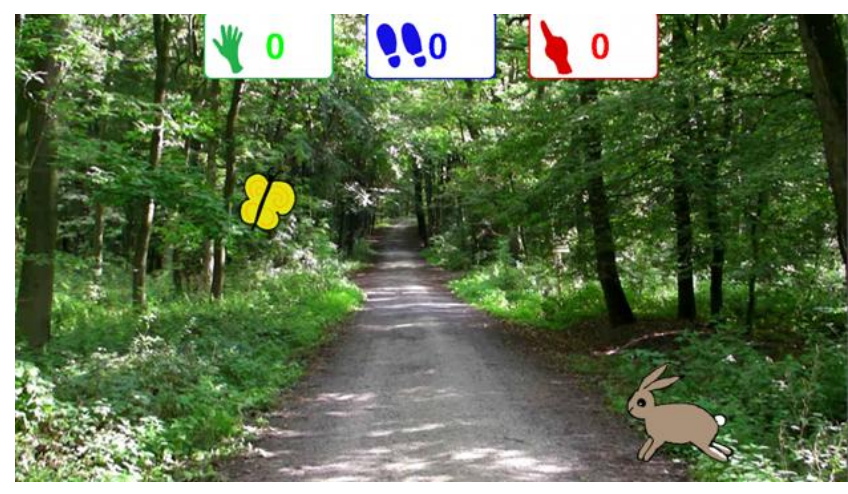

Figure 1. Players are invited to take a virtual walk through a park while competing in small mini-games.

- The walker performs walking movements on the Balance Board to progress through the world.

- $\quad$ The shaker engages in simple counting tasks by shaking the Wii Remote, e.g. following the number of a certain type of game elements.

- The pointer tries to catch in-game items by pointing the Wii Remote at them.

The game features two different multiplayer modes: One can either play together in a cooperative team or one can decide to engage in a competitive game mode.

\subsubsection{Resources}

Players have to focus on the management of personal resources such as cognitive and physical abilities depending on their particular role: First, users generally need to understand the basic flow of the game. Second, during demanding periods of play which present one or more mini games, sustainable attention is required. Third, during periods of retardation and relaxation, the player yet has to be prepared for more challenging situations, thus the user's vigilance is addressed. Finally, the walker continuously needs to step on the balance board in order to allow the team to progress in the game. Regarding exertive aspects, this is the most challenging role and should be picked up by players who are well able to maintain physical exercise throughout play.

\subsubsection{Boundaries}

The boundaries of SilverPromenade are clearly defined: The main action of the game is purely experienced within the virtual context. Whether actions or results of players' engagement in competition mode are discussed among players depends on mutual agreements. Additionally, in-game boundaries are introduced through a limitation of the procedures available to the player: Users cannot leave paths displayed within the game and their impact on mini games is limited to a small set of allowed actions.

\subsubsection{User Interface}

To account for the needs of its target audience, SilverPromenade features the Nintendo Wii Remote and the Nintendo Wii Balance Board as primary input devices: When implemented with the requirements of frail elderly in mind, both controllers are accessible to seniors. Players are free to engage in the game while sitting on a chair or in a standing position. Thereby, individual abilities of the user can be taken into account [8].

Regarding the graphical user interface, SilverPromenade features a linear menu structure which gradually leads the user through the

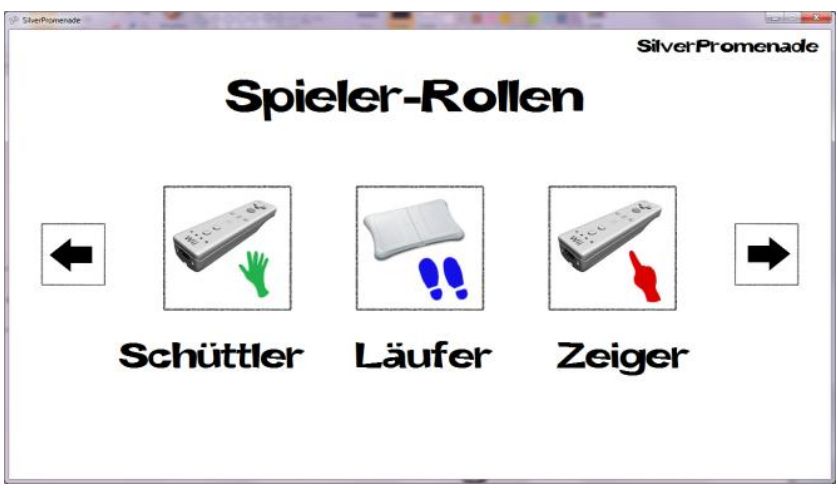

Figure 2. Main menu featuring the selection of player roles: Shaker, walker and pointer.

settings and level selection process (cf. Figure 1). In-game graphics were designed to meet the needs of frail elderly persons: The implementation of visual effects was avoided, relevant game objects are highlighted through contrast and color settings and the virtual world is reduced to video output.

\subsubsection{Core Mechanics}

The core mechanics of SilverPromenade were designed with the special requirements of frail elderly persons in mind. Basically, the game features a drastically reduced level of complexity in order to allow senior citizens without prior gaming experience to engage in play regardless of cognitive or physical impairments: The interaction paradigm associated with the role of the shaker requires the user to quickly move the Wii Remote in order to count certain items displayed within the game and is referred to as shaking. The walker needs to execute alternating pressure on both sides of the board which results in in-game video playback and the illusion of walking through the respective virtual environment. The pointer has to lift the Wii Remote in order to allow for the registration of user input via the infrared interface. If a certain type of interaction is not suitable, the game offers the possibility of deactivating one or more roles in order to reduce the complexity of the game and allow the player to focus on his or her strengths to support a positive gaming experience.

\section{EVALUATION}

In this section, the results of an early evaluation of SilverPromenade are described. The goal of the evaluation was to collect and compare first data of frail elderly living in nursing homes. Previous playing experience was the considered to be an independent variable as some of the seniors had participated in previous gaming sessions and we were interested in performance differences among both groups. The dependent variables game usability and player experience were measured through a questionnaire as well as observations and player performance data.

\subsection{Research Questions}

Generally speaking, only few entertainment systems have targeted frail senior citizens living in nursing homes, thus little data regarding their gaming abilities is available. Therefore, the evaluation tries to examine research questions which may support the future development of digital games for frail elderly persons experiencing age-related changes and impairments. It was designed to examine the following three research questions related to the fields of interface design, game design, and player experience of SilverPromenade: 


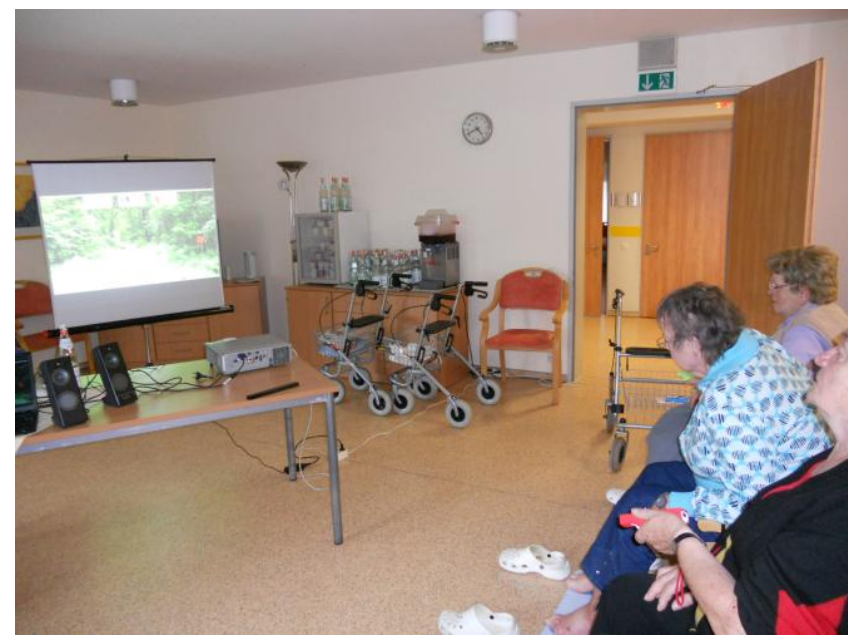

Figure 3. Elderly women immersed in the game.

- Interface design: Is the game usable for frail elderly persons? Is the game interface accessible to elderly despite age-related impairments?

- Player experience: What is the personal experience of players testing SilverPromenade and how do they perceive themselves during play?

- Game design: Which aspects of the prototype were either positively or negatively perceived by the players and therefore might be subject to future improvement or extension?

\subsection{Evaluation Design}

\subsubsection{Participants}

The evaluation of SilverPromenade included two groups of senior citizens and was supported by three nursing homes located in Duisburg, Germany. A total of 18 persons permanently living in full-care nursing homes participated in the evaluation. Fourteen of them were female. While one group of participants (Group 1, $\mathrm{N}=9$ ) also participated in previous gaming sessions [8] and was therefore used to playing video games and familiar with the input devices, particularly the Wii Sports games bowling and tennis as well as Wii Fit balance games. The second group (Group 2, N=9) did not have any prior experience playing digital games. The average age within group 1 was 80 years $(S D=6.89)$, the average age within group 2 was 81 years $(S D=6.21)$. Almost all of the subjects $(\mathrm{N}=15)$ suffered from evident age-related diseases causing decrements in their physical abilities. Except for one person within group 2, all of the participants experienced decrements in sensomotor skills and were dependent on assistive devices for walking. One participant of each group was diagnosed with mild dementia.

\subsubsection{Procedure}

At the beginning of the evaluation, all participants received a short briefing regarding their tasks and the structure of the study and were asked to sign a consent form. Prior to engaging in play, it was verified that none of the participants suffered from diseases which would put them in danger when participating in the evaluation and playing physically challenging games. This was followed by a gaming session during which the participants were invited to play the forest scenario of SilverPromenade. First, each participant was given the opportunity of exploring the game in single player mode while being accompanied by their peers, then subjects were asked to participate in local multiplayer sessions. Each participant was asked to play two levels in each mode. Finally, the subjects were asked to fill out a questionnaire including personal details, questions regarding their personal gaming experience and the usability of SilverPromenade. During this phase, they were assisted by nursing staff if necessary. At the end of the evaluation, we explained the goal of the study and thanked for the participants for their contribution.

\subsubsection{Methodology}

In order to evaluate SilverPromenade, observations were used in combination with a short questionnaire. To provide an objective measure of the player's performance and to support possible findings related to subjective impressions reported through the questionnaire, the collection of player performance data was included in the evaluation. Hence, the player's gaming results were recorded in order to evaluate individual performances.

The questionnaire which was applied during the evaluation was specifically directed towards senior citizens: Besides personal information, it includes a shortened version of the Game Experience Questionnaire [12] and one item from ISO-Norm Questionnaire 9241/10 [24]. The Game Experience Questionnaire (GEQ) measures the dimensions challenge, competence, flow, immersion, negative affect, positive affect and tension. Because of age-related effects on cognitive abilities such as the participant's attention span, the original version of the questionnaire was not suitable due to its length. Based on considerations for the development of a Kids GEQ [23] as well as suggestions regarding the reduction of the GEQ to be used among active seniors [21], a short version of the questionnaire was used to at least screen the above mentioned dimensions. Furthermore, one item of the ISONorm Questionnaire 9241/10 which aims to further examine the usability of the game was introduced in combination with an additional item investigating the accessibility of movements which are required to play the game. Thereby, potential problems regarding the interface design of the game are addressed. In order to facilitate the use of a Likert-scale for possibly cognitively impaired elderly, a smiley-based visualization of its five levels was introduced in addition to the regular scale [13].

Besides the aforementioned instruments, all participants of the evaluation were observed during the gaming session. Special attention was paid to the use of the controllers, the participants' understanding of menu structures as well as in-game behavior. Because SilverPromenade was designed with certain social aspects in mind, the communication between players was observed in order to draw conclusions regarding the interaction between co-players as well as active and passive participants.

\subsection{Results}

\subsubsection{Player Performance Metrics}

This section describes the players' scores which were recorded during the gaming sessions. The scoring was dependent on the player's role: The walker received one point per step; the shaker and pointer were rewarded ten points on successful completion of their task, which occurred less frequently than walking interaction. Data was collected in singleplayer mode with users playing at least two roles depending on their individual abilities, and in multiplayer mode where players only had to execute one task at a time. Due to age-related impairments, only few players were able to perform the role of the walker, drastically reducing the number of participants in that condition for singleplayer $(\mathrm{N}=6)$ and multiplayer mode $(\mathrm{N}=2)$. 
Table 1. Player performance in singleplayer mode.

\begin{tabular}{cccc}
\hline & $\begin{array}{c}\text { Mean score } \\
\text { experienced } \\
\text { players }(S D)\end{array}$ & $\begin{array}{c}\text { Mean score } \\
\text { inexperienced } \\
\text { players }(S D)\end{array}$ & $\begin{array}{c}\text { Mean score } \\
\text { all players } \\
(S D)\end{array}$ \\
\hline Walker & $88.57(18.16)$ & $62.75(6.72)$ & $82.83(19.56)$ \\
\hline Pointer & $88.33(41.53)$ & $54.44(31.37)$ & $71.39(39.73)$ \\
\hline Shaker & $98.89(40.21)$ & $96.67(27.04)$ & $97.78(33.26)$ \\
\hline
\end{tabular}

Table 1 shows the results in singleplayer mode for experienced players and inexperienced players as well as the values for all participants. Likewise, table 2 presents results for multiplayer mode. In both game modes, observed standard deviations are rather large, suggesting differences in players' performances within groups.

In summary, the results gathered from the small sample in this study suggest that experienced participants outperformed inexperienced players on the walker and pointer role. We then analyzed the results for statistical significance using one-factorial ANOVAs. Possibly because of the small sample sizes, almost no statistically significant differences were discovered in the results provided for any of the roles: Solely in multiplayer mode, trained players (group 1) significantly outperformed inexperienced participants when playing the role of the shaker $\left(F_{(1,13)}=5.180\right.$, $p \leq .05$ ). However, a calculation of Cohen's $d$ (cf. [3]) shows large effect sizes (cf. [15]) for the differences between experienced und inexperienced players when playing the roles of the walker $(d=1.89)$ and the pointer $(d=.92)$ in singleplayer mode as well as in multiplayer mode (walker: $d=3.58$, pointer: $d=1.40$ ). In latter mode, the difference between the two groups when playing the shaker seems to be large, too $(d=1.27)$, whereas the shaker scores in singleplayer mode do not seem to differ $(d=.06)$.

\subsubsection{Shortened Game Experience Questionnaire}

Generally, the results of the basic version of the GEQ indicate extremely high scores for items examining positive affect $(M=3.11, S D=1.11)$ and immersion $(M=2.33, S D=1.18)$, while the results for negative affect $(M=0.39, S D=0.85)$ are very low (cf. Figure 4). Values for tension $(M=0.83, S D=1.15)$, challenge $(M=1.17, S D=1.15)$ and flow $(M=1.39, S D=1.09)$ are at a low level while the result for competence $(M=2.17, S D=1.09)$ is relatively high. Additionally, the results show low scores for items exploring the usability $(M=0.83, S D=0.61)$, yet higher ratings when inquiring the accessibility $(M=2.44, S D=1.46)$ of the prototype. Yet, certain differences in ratings between experienced and inexperienced players were observed (cf. Table 3 ).

Table 3. Mean results for the shortened Game Experience Questionnaire.

\begin{tabular}{lccc}
\hline & $\begin{array}{c}\text { Experienced } \\
\text { players }(S D)\end{array}$ & $\begin{array}{c}\text { Inexperienced } \\
\text { players }(S D)\end{array}$ & $\begin{array}{c}\text { All players } \\
(S D)\end{array}$ \\
\hline Tension & $0.89(1.36)$ & $0.78(0.97)$ & $0.83(1.15)$ \\
\hline Challenge & $1.22(1.20)$ & $1.11(1.17)$ & $1.17(1.15)$ \\
\hline Flow & $0.89(0.93)$ & $1.89(1.05)$ & $1.39(1.09)$ \\
\hline Competence & $2.11(0.78)$ & $2.22(1.39)$ & $2.17(1.10)$ \\
\hline Negative Affect & $0.11(0.33)$ & $0.67(1.12)$ & $0.39(0.85)$ \\
\hline Positive Affect & $3.00(0.87)$ & $3.11(1.36)$ & $3.06(1.11)$ \\
\hline Immersion & $2.33(1.23)$ & $2.33(1.23)$ & $2.33(1.89)$ \\
\hline
\end{tabular}

Table 2. Player performance in multiplayer mode.

\begin{tabular}{cccc}
\hline & $\begin{array}{c}\text { Mean score } \\
\text { experienced } \\
\text { players }(S D)\end{array}$ & $\begin{array}{c}\text { Mean score } \\
\text { inexperienced } \\
\text { players }(S D)\end{array}$ & $\begin{array}{c}\text { Mean score } \\
\text { all players } \\
(\text { SD })\end{array}$ \\
\hline Walker & $141.25(30.05)$ & $60.00(11.31)$ & $100.63(50.44)$ \\
\hline Pointer & $141.25(51.05)$ & $83.75(27.50)$ & $112.50(48.84)$ \\
\hline Shaker & $143.57(49.64)$ & $97.14(21.19)$ & $120.36(43.87)$ \\
\hline
\end{tabular}

Comparing groups 1 and 2 again using one-factorial ANOVAs, no significant differences regarding the impact of gaming experience can be reported except for the dimension Flow, where experienced players reported significantly higher results $\left(M_{\mathrm{Gr} 1}=1.89, M_{\mathrm{Gr} 2}=0.89, F_{(1,17)}=4.563, p \leq .05\right)$. A calculation of Cohen's $d$ revealed large differences in reported flow $(d=1.01)$ and perceived usability $(d=.97)$, and a mid-size difference in experienced negative affect between both groups.

\subsubsection{Observations}

During the gaming sessions, participants were observed in order to gain further insight into their interaction with the game. First, their use of the Nintendo Wii Remote and Balance Board controllers was examined. Players without previous gaming experience regarded the input devices as fragile technology, while experienced participants simply picked up the controllers and joined the game. When playing the walker role, participants immediately understood the main idea behind the device, yet many experienced problems executing correct movements: Only one player was able to engage in the game while standing, and seated players experienced difficulties in executing correct movements: Some of the participants shifted their upper body instead of moving their legs, others were unable to execute pressure on one foot only. As pointer, some of the players encountered difficulties when using the Wii Remote as the sensor bar requires the player to point the remote at it directly. Furthermore, they often had to be reminded to use their stronger hand for the pointing mote to facilitate the interaction with the game. However, when engaging in play as shaker, participants generally did not seem to encounter any problems. All of the players required assistance when navigating through the main menu. Yet, most of them were able to read and understand the information presented on different screens and could make most decisions necessary to start a new level.

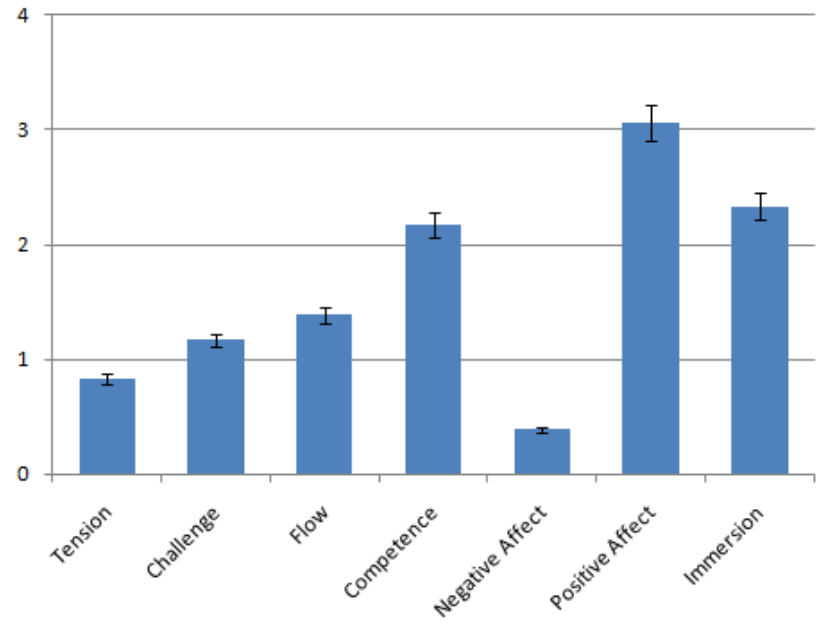

Figure 4. Mean GEQ ratings by all players (CI: 95\%). 
The participants of the evaluation also showed differences in their in-game behavior. Participants with gaming experience were generally familiar with the phenomenal connection between input devices, interface elements and game objects and therefore only required little instruction and support in order to play the game. In contrast, inexperienced participants had to be explained the dependencies between input movements and in-game results due to their lack of gaming experience. In singleplayer mode, participants among both groups showed difficulties multi-tasking while playing several roles. Many participants focused on one role while neglecting others, e.g. stopped walking in order to be able to catch butterflies. In multiplayer mode, the players' comments showed that they found it easier to complete their tasks than in single player mode. Comments among players showed that the theme of the game was perceived positively, e.g. one player stated: "I like to see all the green of the forest again, it's been so long since I last went there." Also, including animals as main characters of the mini games was perceived well by the audience, often making remarks about them, such as "Look, the mean little rabbit is back!" or "We need to catch that butterfly over there!".

Generally, vivid communication between players was observed during the gaming sessions. Once a level was started, many of the elderly persons present engaged in passive play by supporting the active players by trying to identify different game objects such as the rabbit or butterfly. Comments like "There, the rabbit appeared, it's on the left!" or "Move up quickly to catch the butterfly!" were frequently made. Furthermore, active players helped each other by passing on knowledge regarding the interaction with the game and giving hints once relevant game objects appeared on screen. Most importantly, participants of the evaluation were also observed discussing and comparing their scores at the end of the playing sessions, engaging with their peers and extending interaction beyond their participation in play.

\subsection{Discussion}

The overall findings of the evaluation suggest that a game like SilverPromenade offers a demanding, yet enjoyable gaming experience for frail elderly players. This is supported by the analysis of player performance metrics which implies that the game appeals to a large group of senior citizens regardless of agerelated impairments. Although SilverPromenade generally seems to be accessible to persons without prior gaming experience, the evaluation results suggest effects of training on player performance and experience. Additionally, the evaluation also revealed certain problems concerning game control for elderly, particularly influenced by individual age-related impairments.

The fact that experienced players generally outperformed users who had never played video games before shows that previous gaming experience may affect player performance, suggesting that elderly players are able to learn how to interact with new technologies despite the possible impact of age-related difficulties in motor learning. Differences in flow measured among both player groups might be attributed to the fact that players without prior gaming experience seemed to encounter more problems when interacting with the game, which is supported by a lower level of perceived usability and an increase of negative affect.

Regarding the hardware interface of the game, the evaluation showed that the Wii Remote may well be used by older adults if designers avoid its small buttons and time-based interaction sequences. The Balance Board was also accessible to most participants, yet individual impairments should be accounted for when including foot-based input in games for frail elderly. While the information presented by the in-game GUI was understood by all players, further research regarding the design of graphical user interfaces for frail elderly without prior gaming experience is necessary to allow for an independent setup of gaming sessions.

Observations and player scores suggest that the interaction paradigms associated with the pointer and walker roles have to be improved to account for severe age-related changes: When faced with pointing tasks, the Wii Remote needs to be held steadily to face the sensor bar at all times, hence players suffering from decrements in motor control were especially disadvantaged. The walker role might be improved by offering different interaction paradigms, thus players with insufficient motor skills could be offered the opportunity of executing repeated pressure on both feet instead of alternating movements.

Furthermore, observations during singleplayer mode suggest that the cognitive load of managing two or more player roles simultaneously was too high for many of the participants, an aspect which proved to be well manageable for younger players in previous tests. Thus, a reduced game design allowing the players to focus on one main task might be beneficial. In this context, the local multiplayer mode was a valuable alternative: Players were faced with one challenge only, and several persons were given the possibility of engaging in play and supporting each other.

In the context of this evaluation, the application of a shortened version of the Game Experience Questionnaire was successful in terms of screening game experience among frail elderly persons. Despite age-related cognitive and physical changes, all of the participants were able to understand and answer all questions, while the reduced length fit their attention span. Yet, further research regarding test criteria like reliability and internal consistency of such a shortened version is necessary.

\section{CONCLUSION AND FUTURE WORK}

In this paper, the design of video games for senior citizens was discussed focusing on frail elderly persons. The game SilverPromenade accounts for the impact of age on various levels. It introduces simplistic interaction paradigms and a minimalistic, senior-friendly gameplay based on the Nintendo Wii Remote and Wii Balance Board which allow for the participation of both impaired and active senior citizens in play. The evaluation showed that elderly persons enjoy engaging in a digital game and results indicate that the game prototype SilverPromenade is accessible to a broad audience. In the context of the evaluation, a combination of observations, game metrics and a senior-friendly questionnaire was suggested as research tool when evaluating digital games with frail elderly participants.

\subsection{Conclusion}

On a general level, the conceptual design, development and evaluation of the game prototype SilverPromenade has shown that frail elderly enjoy playing digital games despite age-related cognitive or physical impairments and changes. Furthermore, the evaluation results suggest that many aspects of the game prototype met the needs of an elderly audience in terms of interaction design and the complexity of game mechanics. Based on the results described within this paper, the following three design opportunities for game designers could be identified:

Carefully explore the abilities of your target audience to provide accessible gameplay. Despite a thorough examination of common age-related changes and the adaption of SilverPromenade, players experienced difficulties interacting with the game due to 
individual impairments. To address this issue, we recommend a participatory, highly iterative design approach which allows elderly persons to test and comment on early interaction prototypes to improve game accessibility. Participatory game design with elderly has successfully been implemented to create game concepts [29], and we believe that it offers a great potential for designing senior-friendly interaction in games.

Create meaningful metaphors and relate to real-world actions to facilitate the entry into play. Elderly persons frequently lack gaming experience [22], and the evaluation of SilverPromenade has shown that game mechanics and interaction paradigms related to well-known real-world actions largely facilitated elderly persons' entry into play. Considering this issue during the design process might foster the creation of accessible, easy to learn games for elderly.

Provide games which adapt to a broad range of players and gaming situations. Besides the creation of adaptable game interfaces, providing different player roles and levels of game complexity is crucial when addressing frail elderly. Also, the gaming situation of senior citizens living in nursing homes has to be considered: Games are likely to be installed in public spaces, hence supporting a variable number of players as well as accounting for passive players and bystanders is important. To allow for unaccompanied gaming sessions, ad-hoc play without extensive setup routines should be explored.

Also, the evaluation process proved to be challenging since such a diverse target audience largely unfamiliar with technical vocabulary had to be addressed. In addition, cognitively and physically impaired persons joined each other in play and often required assistance apart from issues directly related to the game. It is also important to keep in mind that future generations of senior citizens will be familiar with digital games due to generational differences: Teenagers and young adults of today have grown up playing digital games and are likely to continue engaging with this medium. Thus, the considerations regarding game design for elderly presented within this paper are certainly valid for a vast majority of today's senior citizens, whereas the elderly population of the future may be able to engage in more complex video games due to a lifelong gaming history and extensive domain knowledge.

\subsection{Future Work}

Future work on SilverPromenade includes the improvement of the pointer and walker roles in order to account for a wider range of age-related needs. Additionally, more challenging mini games will be included for experienced players, for instance game mechanics which might require the player to recognize multiple objects or select a specific object from a group (e.g. picking a red rabbit out of a group of colorful ones). Furthermore, mini games requiring all players to act together might be introduced in order to further address cooperation and communication between players to evaluate possible effects of games on social interaction between elderly persons.

The evaluation also revealed certain challenges regarding the creation of intuitive input mechanisms, suggesting a need for additional research regarding the design of senior-friendly interaction paradigms for entertainment systems. In the context of game design for seniors living in nursing homes, the development of exergames to encourage physical activity might be one of many valuable design opportunities. In this context, the further exploration of full-body interfaces such as Microsoft's Kinect might provide designers with suitable input devices. Additionally, research regarding possible benefits of playing digital games, e.g. games for brain training or practicing physical abilities, is necessary with a focus on geriatric and medical aspects. Also, the further investigation of intergenerational play is promising as the creation of games which address different age groups might offer a new space for old and young members of our society.

Finally, the results of the evaluation have to be set into perspective regarding the situation of inhabitants of full-care nursing homes. Although they are offered a variety of leisure activities, it is well possible that the novelty of digital games and the presence of external guests have positively influenced the seniors' perception of SilverPromenade. Hence, to draw conclusions regarding the general attractiveness of digital games as leisure activity, further research with a focus on the long-term development of player motivation over several weeks is necessary.

Generally speaking, one of the most powerful aspects of game development for elderly living in nursing homes is the opportunity of raising their quality of life by providing them with a diverse set of leisure activities. Video games offer the possibility of encouraging seniors to remain active even when living in full-care environments. In this context, it is important to provide technology which is easy to set up in order to relieve nursing staff and reduce access barriers. Apart from economic advantages, this is an aspect which will hopefully be recognized by the games industry, as an increase of professional approaches towards game design for elderly might also largely increase the number and quality of games available on the market. Finally, this might encourage more nursing homes to enrich the lives of their guests by introducing suitable digital games.

\section{REFERENCES}

[1] Adams, E. 2010. Fundamentals of Game Design. Berkeley: New Riders Publishing.

[2] Burke, J.W., McNeill, M.D.J., Charles, D.K., Morrow, P.J., Crosbie, J.H., \& McDonough, S.M. 2009. Optimising engagement for stroke rehabilitation using serious games. In: The Visual Computer: International Journal of Computer Graphics. Serious Games and Virtual Worlds, 1085-1099.

[3] Cohen, J. 1988. Statistical power analysis for the behavioral sciences (2nd ed.). New Jersey: Lawrence Erlbaum.

[4] Czaja, S. J., \& Lee, C. C. 2008. Information Technology and Older Adults. In: Sears, A. \& Jacko, J.A. (Eds.): The Human Computer Interaction Handbook. New York, New York, USA: Lawrence Erlbaum Associates.

[5] Flores, E., Tobon, G., Cavallaro, E., Cavallaro, F. I., Perry, J. C., Keller, T. 2008. Improving patient motivation in game development for motor deficit rehabilitation. Proceedings of ACE '08, Yokohama, Japan.

[6] Fullerton, T. 2008. Game Design Workshop, Second Edition: A Playcentric Approach to Creating Innovative Games. Burlington: Morgan Kaufmann Publishers.

[7] Gamberini, L., Alcaniz, M., Barresi, G., Fabregat, M., Prontu, L., \& Seraglia, B. 2008. Playing for a Real Bonus: Videogames to Empower Elderly People. Journal of CyberTherapy \& Rehabilitation, 1(1) 2008, 37-48.

[8] Gerling, K. \& Masuch, M. 2011. When Gaming is not Suitable for Everyone: Playtesting Wii Games with Frail 
Elderly. $1^{\text {st }}$ Workshop on Game Accessibility: Xtreme Interaction Design (GAXID'11), Bordeaux, France.

[9] Gerling, K.M., Schild, S. \& Masuch, M. 2010. Exergame Design for Elderly Users: The Case Study of SilverBalance. Proceedings of $A C E$ '10, Taipei, Taiwan.

[10] Hanneton, S. \& Varenne, A. 2009. Coaching the Wii. IEEE International Workshop on Haptic Audio Visual Environments and Games, 2009.

[11] Ijsselsteijn, W., Nap, H.H. \& de Kort, Y. 2007. Digital Game Design for Elderly Users. Proceedings of Futureplay 2007, Toronto, Canada.

[12] Ijsselsteijn, W.A., de Kort, Y.A.W., and Poels, K. (in preparation). The Game Experience Questionnaire: Development of a self-report measure to assess the psychological impact of digital games.

[13] Jäger, R. 2004. Konstruktion einer Ratingskala mit Smilies als symbolische Marken. Diagnostica, 50(1), 31-38.

[14] Jung, Y., Li, K.J., Janissa, N.S., Gladys, W.L.C. \& Lee, K.M. 2009. Games for a Better Life: Effects of Playing Wii Games on the Well-Being of Seniors in a Long-Term Care Facility. Proceedings of IE 2009.

[15] Kenny, D.A. 1987. Statistics for the social and behavioral sciences. Boston: Little, Brown.

[16] Keyani, P., Hsieh, G., Mutlu, B., Easterday, M., \& Forlizzi, J. 2005. DanceAlong: Supporting Positive Social Exchange and Exercise for the Elderly Through Dance. Proceedings of the annual SIGCHI Conference on Human Factors in Computing Systems, Portland, Oregon, USA.

[17] Khoo, E.T. \& Cheok, A.D. Age Invaders: Inter-generational Mixed Reality Family Game. The International Journal of Virtual Reality, 5(2): 45-50.

[18] Lee, Y.S., Basapur, S., Chaysinh, S., \& Metcalf, C. 2011. Senior Wellness: Pracitices of Community Senior Centers. Proceedings of the 28th of the international conference extended abstracts on Human factors in computing systems, Vancouver, BC, Canada.

[19] Mahmud, A.A., Mubin, O., Shahid, S., \& Martens, J. 2008. Designing and evaluating the tabletop game experience for senior citizens. Proceedings of NordiCHI '08.

[20] Mubin, O., Shahid, S. \& Mahmud, A.A. 2008. Walk 2 Win: Towards Designing a Mobile Game for Elderly's Social Engagement. Proceedings of the 22nd Annual British HCI Conference, Liverpool, England.
[21] Nacke, L., Nacke, A. \& Lindley, C. 2009. Brain Training for Silver Gamers: Effects of Age and Game Form on Effectiveness, Efficiency, Self-Assessment, and Gameplay Experience. CyberPsychology \& Behavior, 12(5): 493-499.

[22] Nap, H.H., de Kort, Y.A.W., \& Ijsselsteijn, W.A. 2009. Senior gamers: preferences, motivations and needs. Gerontechnology, 8(4), 247-262.

[23] Poels, K., Ijsselsteijn,W. \& de Kort, Y. 2008. Development of the Kids Game Experience Questionnaire. Proceedings of Meaningful Play 2008, East Lansing, USA.

[24] Pruemper, J. 2010. Fragebogen ISONORM. Available at http://www.ergo-online.de/site.aspx?url=html/software/verfa hren_zur_beurteilung_der/fragebogen_isonorm_online.htm, last access: 14.10.2010.

[25] De Schutter, B. \& Vanden Abeele, V. 2008. Meaningful Play in Elderly Life. Proceedings of the 58th Annual Conference of the International Communication Association, Montreal, Canada.

[26] Shim, N., Baecker, R., Birnholtz, J. \& Moffatt, K. 2010. TableTalk Poker: An Online Social Gaming Environment for Seniors. Proceedings of FuturePlay 2010, Vancouver, BC, Canada

[27] SilverFit B.V. 2011. SilverFit. Available at http://www.silverfit.nl, last access: 22/05/2011.

[28] Theng, Y., Dahlan, A. B., Akmal, M. L., \& Myint, T. Z. 2009. An exploratory study on senior citizens' perceptions of the Nintendo Wii. Proceedings of ICREATE '09, 5, 1. New York, New York, USA: ACM Press.

[29] Vanden Abeele, V. and Van Rompaey, V. 2006. Introducing Human-Centered Research to Game Design: Designing Game Concepts for an with Senior Citizens. Extended abstracts of the annual SIGCHI conference on Human factors in computing, Montreal, Quebec, Canada.

[30] Vanderheiden, G.C. 1997. Design for People with Functional Limitations Resulting from Disability, Aging, or Circumstance. In: Salvendy, G. (Ed.): Handbook of Human Factors and Ergonomics. New York: John Wiley and Sons.

[31] Weisman, S. 1983. Computer Games for the Frail Elderly. Gerontologist, 23(4): 361-363.

[32] Young, W., Ferguson, S., Brault, S., \& Craig, C. 2010. Assessing and training standing balance in older adults: A novel approach using the 'Nintendo Wii' Balance Board. Gait \& Posture, in print. 